Document downloaded from:

http://hdl.handle.net/10251/83202

This paper must be cited as:

Pitarch Pérez, JL.; Sala Piqueras, A. (2016). Control synthesis for polynomial discrete-time systems under input constraints via delayed-state Lyapunov functions. International Journal of Systems Science. 47(5):1176-1184. doi:10.1080/00207721.2014.915357.

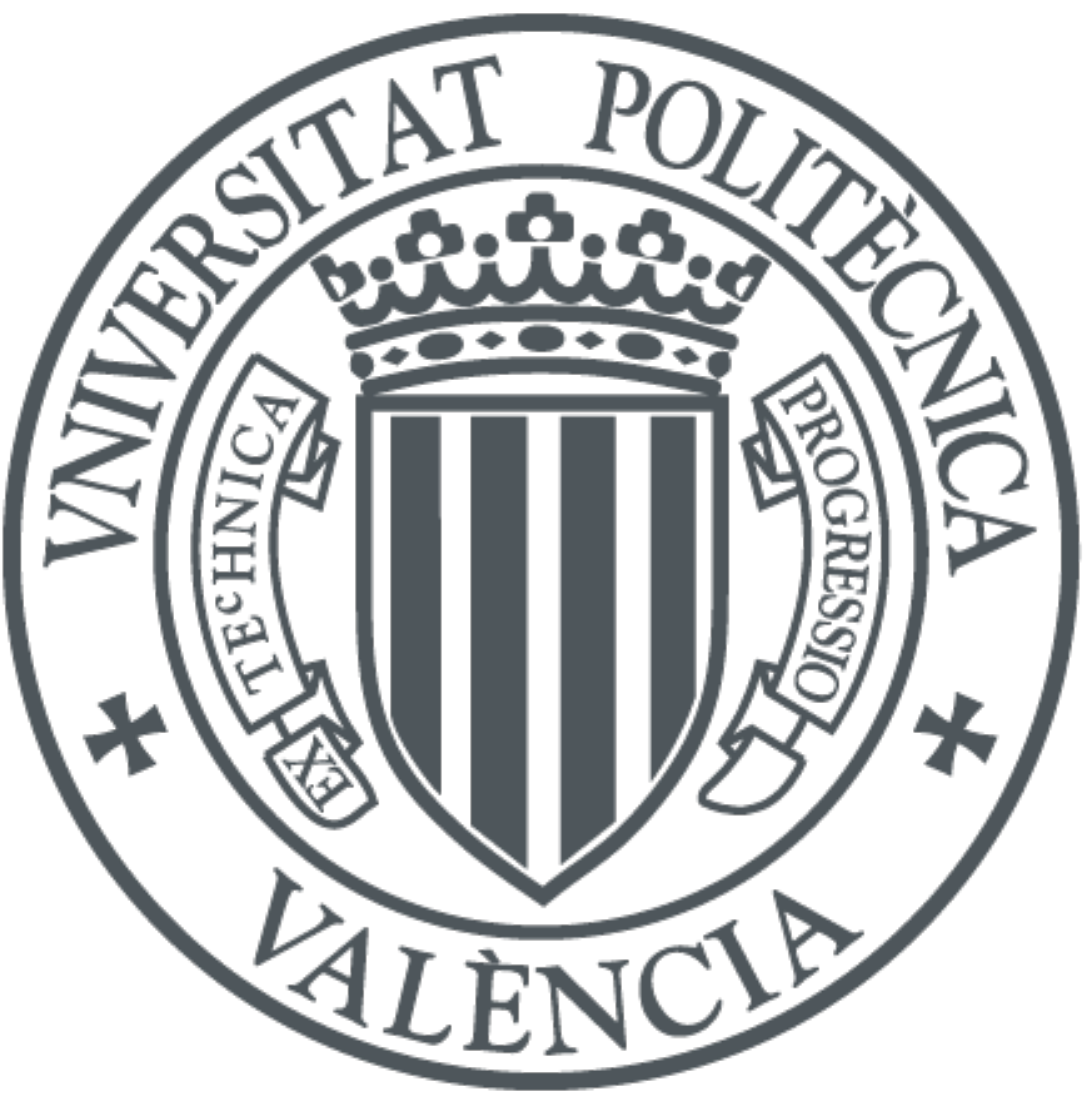

The final publication is available at

http://doi.org/10.1080/00207721.2014.915357

Copyright Taylor \& Francis

Additional Information 


\title{
Control synthesis for polynomial discrete-time systems under input constraints via delayed-state Lyapunov functions
}

\author{
J.L. Pitarch ${ }^{* a}$, A. Sala ${ }^{\mathrm{a}}$, J. Lauber ${ }^{\mathrm{b}}$ and T.M. Guerra ${ }^{\mathrm{b}}$ \\ ${ }^{*}$ Corresponding author. \\ ${ }^{a}$ Instituto Univ. de Automática e Informática Industrial (AI2), Universitat Politécnica \\ de Valencia, Spain.
}

${ }^{b}$ LAMIH UMR CNRS 8201, Université de Valenciennes et du Hainaut-Cambrésis, France.

E-mail : jopipe1@ upvnet.upv.es (J.L.Pitarch), asala@isa.upv.es (A.Sala), jlauber@univ-valenciennes.fr (J.Lauber), thierry.guerra@univ-valenciennes.fr (T.M.Guerra).

- This article has been accepted for publication in International Journal of Systems Science http://dx.doi.org/10.1080/00207721.2014.915357

- PUBLISHER $=\{$ Taylor and Francis $\}$,

- VOLUME $=\{47\}$, ISSUE $=\{5\}$, ISSN $=\{0020-7721\}$, PAGES $=\{1176-1185\}$, 


\title{
Control synthesis for polynomial discrete-time systems under input constraints via delayed-state Lyapunov functions
}

\begin{abstract}
This paper presents a discrete-time control design methodology for inputsaturating systems using a Lyapunov function with dependence on present and past states. The approach is used to bypass the usual difficulty with full polynomial Lyapunov functions of expressing the problem in a convex way. Also polynomial controllers are allowed to depend on both present and past states. Furthermore, by considering saturation limits on the control action, the information about the relationship between the present and past states is introduced via Positivstellensatz multipliers. Sum-of-squares (SOS) techniques and available SDP software are used in order to find the controller.
\end{abstract}

Keywords: delayed Lyapunov function, discrete time, polynomial systems, control design, SOS approach, stabilization, convex optimization.

\section{Introduction}

Many smooth nonlinear systems can be transformed to polynomial ones, either by a Taylor-series approximation (Sala and Ariño 2009) or by a change of variable and state augmentation (Papachristodoulou and Prajna 2005). As convex programming tools for polynomial systems have been recently developed (see references below), the polynomial approach may be used to obtain alternative nonlinear control solutions to others in literature (Slotine 1991, Khalil 2002, Koshkouei and Burnham 2011), based on a systematic modelling and convex-programming approach. The approach, however, involves some conservative choices in order to get a reasonable computational cost: finite degree of Lyapunov functions and finite degree and number of KKT-like multipliers associated to algebraic constraints (Jarvis-Wloszek et al. 2005).

Stability analysis and control design for polynomial systems has received attention in 
recent literature, both in continuous-time (Pozo and Rodellar 2010, Chesi 2011) and discrete-time settings (Xu et al. 2007, Tanaka et al. 2008). The basic framework uses Sum of Squares (SOS) techniques (Balas et al. 2012, Seiler et al. 2013) and Positivstellensatz theorems (Jarvis-Wloszek et al. 2005) in order to prove local stability. The reader is referred to Chesi (2010) for a survey and additional literature regarding the main ideas in the approach.

In this polynomial control framework, if the controller and a Lyapunov function have to be simultaneously found, the discrete-time design case usually leads to a non-convex problem which has to be solved by $\mathrm{V}-\mathrm{K}$ iterations or any other similar algorithms $(\mathrm{Xu}$ et al. 2007). In order to avoid this problem, Prajna, Papachristodoulou \& Wu (2004); Tanaka et al. (2008) proposed restricting the dependence of the Lyapunov function to the subset $\tilde{x}$ of the states which are not directly affected by the control action (i.e., $\tilde{x}_{k+1}$ does not depend on $u_{k}$ ). This outperforms the classical quadratic case control design but it is still quite restricted.

In this work, the stabilization problem for polynomial systems with input bounds is addressed in a convex way, using the whole state information. The main idea is introducing delayed states in the Lyapunov function which breaks up some bilinear terms and also provides the state-feedback controller with extra degrees of freedom (rationally depending on present and past state values). The use of Lyapunov functions with dependence on delayed scheduling parameters has been successfully applied in the TakagiSugeno LMI framework (Guerra et al. 2012). In the discrete-time case here considered, due to the construction of the involved matrices, there is no need of Krasovskii-like terms in Lyapunov functions, as other developments need (Gassara et al. 2014).

In this paper, the delay idea is applied to include a full delayed state in polynomial sys- 
tems. Information about the relationship between present and past state values is introduced by specifying bounds in the control action and Positivstellensatz multipliers. The approach improves over existent ones in literature, if restricted to convex optimization setups. The recent work Valmorbida et al. (2013) proposes a similar development addressing the polynomial control synthesis for discrete-time systems under actuator saturation. However, their proposal leads to a non-convex bilinear matrix inequality problem, which needs to be solved iteratively without guarantees of global optimality, as widely known (Fukuda and Kojima 2001). Intentionally, analysis and comparison with BMI approaches has been left out of the scope of this paper, because the BMI results depend on the initial conditions and iteration step sizes.

The objective of the paper is, hence, lifting conservativeness in polynomial control by using delayed-state Lyapunov functions and saturation bounds while keeping the resulting SOS conditions convex.

The structure of the paper is as follows: next Section states the notation followed in the rest of the paper as well as summarizes the existent preliminary results related to the current issue and presents the problem statement, Section 3 presents a convex control design methodology by delayed polynomial Lyapunov functions, Section 4 shows an academic example demonstrating the effectiveness of the proposed approach and, finally, a conclusions Section closes the paper.

\section{Preliminaries and notation}

Let us first introduce some notation and basic sum-of-squares results to be used throughout the paper. The set of polynomials in a variable $x \in \mathbb{R}^{n}$ will be denoted by $\mathcal{R}_{x}$, and the $n$-dimensional vectors of polynomials in $x$ as $\mathcal{R}_{x}^{n}$. The corresponding element of a polynomial symmetric expression will be denoted as $(*)$. The frontier of a 
semi-algebraic region $\Omega$ will be denoted by $\partial \Omega$.

Polynomials in variables $x$ which can be decomposed as a sum of squares of other polynomials (SOS) will be denoted by $\Sigma_{x}$ and the $N \times N$ SOS polynomial matrices in $x$ (see Proposition 1) by $\Sigma_{x}^{N}$. SOS decompositions of (matrix) polynomials can be found by searching for a positive semi-definite scalar matrix using SDP software:

Proposition 1 (Scherer and Hol 2005). Let $F(x)$ be an $N \times N$ symmetric polynomial matrix of degree $2 d$ in $x \in \mathbb{R}^{n} . F(x)$ is a SOS polynomial matrix if and only if there exist a constant matrix $Q \geqslant 0$ satisfying

$$
F(x)=(I \otimes z(x))^{T} Q(I \otimes z(x)) \forall x \in \mathbb{R}^{n}
$$

with $z(x)$ being a column vector whose entries are all monomials in $x$ with degree no greater than $d$.

Evidently, SOS polynomials in independent variables $x$ are non-negative, and SOS polynomial matrices are positive semi-definite matrices, for all values of $x$. Note also that the condition in Proposition 1 can be cast as a non-strict linear matrix inequality (LMI) in the elements of $Q$.

The notation used in Jarvis-Wloszek et al. (2005) will be also used in the rest of the paper: given polynomials $\left\{f_{1}, \ldots, f_{t}\right\}, \mathcal{M}\left(f_{1}, \ldots, f_{t}\right)$ will denote the multiplicative monoid, $\wp\left(f_{1}, \ldots, f_{t}\right)$ denotes the cone and $\mathcal{J}\left(f_{1}, \ldots, f_{t}\right)$ denotes the ideal generated by the set of $f_{k}{ }^{\prime} s$. Denote also by $\mathcal{J}^{N}\left(f_{1}, \ldots, f_{t}\right)$ the set of $N \times N$ matrices whose elements belong to the ideal $\mathcal{J}\left(f_{1}, \ldots, f_{t}\right)$. Denote also by $\wp^{N}\left(f_{1}, \ldots, f_{t}\right)$ the subset of $\mathcal{J}^{N}\left(f_{1}, \ldots, f_{t}\right)$ formed by the cone of matrices which are positive semi-definite for any values of the argument variables. 
Using the above notation, the cited work recasts the so-called Positivstellensatz theorem (Stengle 1974) in order to assert local non-negativeness of polynomials in a region with polynomial boundary. The lemma below generalises the concept to local positive semidefiniteness of polynomial matrices:

Lemma 1. The polynomial matrix $P(x) \in \mathcal{R}_{x}^{N \times N}$ is positive semi-definite in a region $\Omega=\left\{x: g_{i}(x)>0, h_{j}(x)=0, i: 1, \ldots, r, j: 1, \ldots v\right\}$ if there exist polynomial matrices $G(x) \in \wp^{N}\left(g_{1}, \ldots, g_{r}\right)$ and $H(x) \in \mathcal{J}^{N}\left(h_{1}, \ldots, h_{v}\right)$ which verify:

$$
P(x)-G(x)+H(x) \in \Sigma_{x}^{N}
$$

Proof: Multiplying (2) by auxiliary variables $v \in \mathbb{R}^{N}$ on the left and right, it results in a polynomial sum-of-squares condition $v^{T}(P(x)-G(x)+H(x)) v \in \Sigma_{x, v}$ so that, if it holds, $v^{T} P(x) v$ is nonnegative in $\Omega$ as required.

Note that computational checking of (2) can be done with the LMI's deriving from Proposition 1. For instance, one choice of matrices above for computations may be

$$
G(x)=\sum_{i=1}^{r} S_{i}(x) g_{i}(x) \quad H(x)=\sum_{j=1}^{v} Z_{j}(x) h_{j}(x)
$$

where $S_{i}(x)$ are SOS matrices and $Z_{j}(x)$ are arbitrary ones, both with unknown coefficients. $S_{i}(x)$ and $Z_{j}(x)$ can be full polynomial matrices or, for instance, only diagonal ones depending on the available computing resources.

Stability of polynomial systems. Consider a polynomial discrete-time system in the form

$$
x_{k+1}=A\left(x_{k}\right) z\left(x_{k}\right)+B\left(x_{k}\right) u_{k}
$$

where $x_{k} \in \mathbb{R}^{n}, u_{k} \in \mathbb{R}^{c}$ are the state vector and control input vector at time instant $k$ 
respectively, $A\left(x_{k}\right) \in \mathcal{R}_{x_{k}}^{n \times m}$ and $B\left(x_{k}\right) \in \mathcal{R}_{x_{k}}^{n \times c}$ are polynomial matrices and $z\left(x_{k}\right) \in$ $\mathcal{R}_{x_{k}}^{m}$ is a polynomial vector in the states. On the sequel, shorthand $z_{k}=z\left(x_{k}\right)$ will be used for brevity.

Define a candidate Lyapunov function $V: \mathcal{D} \rightarrow \mathbb{R}$ as

$$
V\left(x_{k}\right)=z_{k}^{T} Q^{-1}\left(x_{k}\right) z_{k}
$$

where $\mathcal{D} \in \mathbb{R}^{n}$ is an open set, $0 \in \mathcal{D}$ and $Q\left(x_{k}\right) \in \mathcal{R}_{x_{k}}^{m \times m}$ is a polynomial matrix in the states. Note that $Q^{-1}\left(x_{k}\right)$ appears in the Lyapunov function instead of $Q\left(x_{k}\right)$ in order to adapt the standard technique of change of variables for control in LMI framework (Bernussou et al. 1989) to polynomial cases, setting $\rho=Q^{-1}\left(x_{k}\right) z_{k}, V=\rho^{T} Q\left(x_{k}\right) \rho$. Consider now a state-feedback controller in the form

$$
u_{k}=-K\left(x_{k}\right) z_{k}
$$

where $K\left(x_{k}\right)=M\left(x_{k}\right) Q^{-1}\left(x_{k}\right)$ is the feedback gain and $M\left(x_{k}\right) \in \mathcal{R}_{x_{k}}^{c \times m}$. According to Lyapunov theory, the controller (5) stabilizes the system (3) if conditions

$$
\begin{gathered}
V(0)=0 \\
V\left(x_{k}\right)>0, \forall x_{k} \in \mathcal{D}, x_{k} \neq 0 \\
\Delta V=V\left(x_{k+1}\right)-V\left(x_{k}\right) \leq 0,\left\{x_{k+1}, x_{k}\right\} \in \mathcal{D}
\end{gathered}
$$

are satisfied (Khalil 2002). Further if the inequality (8) is strict for $x_{k} \in \mathcal{D} \backslash\{0\}$, then the system is asymptotically stable. Moreover, if $\mathcal{D}=\mathbb{R}^{n}$, stability is global.

In the controller synthesis problem (i.e., the controller has to be found simultaneously with the Lyapunov function), some conservative assumptions are addressed in literature 
(Xu et al. 2007, Tanaka et al. 2008) in order to cast the problem in a convex way:

- If $Q$ is constant and $z_{k}=x_{k}$, the controller synthesis problem becomes convex by Schur complement, resulting in finding $Q$ and coefficients of polynomials in $M\left(x_{k}\right)$ such that, for an arbitrary $\epsilon>0$ :

$$
\left[\begin{array}{cc}
Q & (*)^{T} \\
A\left(x_{k}\right) Q-B\left(x_{k}\right) M\left(x_{k}\right) & Q
\end{array}\right]-\epsilon I \in \Sigma_{x_{k}}^{2 m}
$$

- Following the idea introduced in continuous-time in Prajna, Papachristodoulou \& Wu (2004), consider a Lyapunov function defined by $Q\left(\tilde{x}_{k}\right)$, where $\tilde{x}_{k}=$ $E x_{k} \in \mathbb{R}^{L}$, being $E$ a constant matrix fulfilling ${ }^{1} E B\left(x_{k}\right)=0$. If $z_{k}$ can be expressed as

$$
z_{k}=T\left(\tilde{x}_{k}\right) x_{k}
$$

with $T\left(\tilde{x}_{k}\right) \in \mathcal{R}_{\tilde{x}_{k}}^{m \times n}$, the problem is still convex.

If the above problems render infeasible, local stability conditions can be posed based on modifying conditions (7) and (8) in order to make them hold locally in a so-called region of interest $\Omega \subset \mathbb{R}^{n}$. Lemma 1 enables checking such conditions with SOS programming (sufficient conditions). For instance, the local stability results in $\mathrm{Xu}$ et al. (2007) can be adapted to the notation here as follows:

Corollary 1. If polynomial matrices $G\left(x_{k}\right), H\left(x_{k}\right)$ as defined in Lemma 1 can be found fulfilling

\footnotetext{
${ }^{1}$ A particular case (Tanaka et al. 2008) is choosing $E$ to be a row-selector matrix extracting the state variables whose corresponding row of $B(x)$ is zero (i.e. $\tilde{x}$ are states that don't directly depend on the control input).
} 


$$
\left[\begin{array}{cc}
Q & (*)^{T} \\
A\left(x_{k}\right) Q-B\left(x_{k}\right) M\left(x_{k}\right) & Q
\end{array}\right]-\epsilon I-G\left(x_{k}\right)+H\left(x_{k}\right) \in \Sigma_{x_{k}}^{2 m}
$$

with $\epsilon>0$, then $\Delta V\left(x_{k}\right)$ is locally negative in a region of the state space $\Omega$ except at the origin.

When conditions (6), (7) and (8) hold for all $x \in \Omega$, the system is said to be locally stable in $\Omega$, implying that all level sets $\{x: V(x) \leq \gamma\} \subset \Omega$ are invariant (Khalil 2002). SOS procedures also allow expanding the proven domain of attraction to sets larger than the referred level sets (Pitarch et al. 2013).

Problem statement. The nonlinear nature of (4) and (8) is a fundamental difficulty with non-quadratic Lyapunov functions in discrete-time systems. Up to the authors' knowledge, the general problem of finding a Lyapunov function $Q\left(x_{k}\right)$ and a controller gain $K\left(x_{k}\right)$ together has not been posed in convex form. The Lyapunov functions with dependence on delayed scheduling parameters in Guerra et al. (2012), inspired using a full delayed state polynomial Lyapunov function to reduce the conservativeness of the above results, as discussed next.

\section{Main result}

Consider a delayed-rational candidate Lyapunov function $V\left(x_{k}, x_{k-1}\right)$ in the form

$$
V\left(x_{k}, x_{k-1}\right)=z_{k}^{T} Q^{-1}\left(\tilde{x}_{k}, x_{k-1}\right) z_{k}
$$

and a state-feedback control law which can depend on present and past states

$$
u_{k}=-K\left(x_{k}, x_{k-1}\right) z_{k}
$$

where, $K\left(x_{k}, x_{k-1}\right)=M\left(x_{k}, x_{k-1}\right) Q^{-1}\left(\tilde{x}_{k}, x_{k-1}\right)$, being $Q\left(\tilde{x}_{k}, x_{k-1}\right) \in \mathcal{R}_{\tilde{x}_{k}, x_{k-1}}^{m \times m}$ and $M\left(x_{k}, x_{k-1}\right) \in \mathcal{R}_{x_{k}, x_{k-1}}^{c \times m}$. It will be assumed that there exists a constant matrix $E \in \mathbb{R}^{L \times n}$ 
such that $z_{k}$ can be expressed as (9) and another constant matrix $E^{\perp}$ such that $E^{T} E^{\perp}=$ 0 and the rows of $E$ and $E^{\perp}$ form a basis of $\mathbb{R}^{n}$. Obviously, by definition, the columns of $B$ belong to the row space of $E^{\perp}$.

Consider a region $\Omega$ of the augmented state space:

$$
\begin{gathered}
\Omega_{0}=\left\{x: z(x)^{T} U z(x) \leq R^{2}\right\} \\
\Omega=\left\{x_{k}, x_{k-1}: x_{k} \in \Omega_{0}, x_{k-1} \in \Omega_{0}\right\}
\end{gathered}
$$

and a second region $\Phi, \Phi \subset \Omega$, where initial conditions are supposed to lie in, described as

$$
\Phi=\left\{x_{0}, x_{-1}: \max \left(z_{0}^{T} Y z_{0}, z_{-1}^{T} Y z_{-1}\right) \leq \beta^{2}\right\}
$$

here $U$ and $Y$ are constant user-defined matrices with suitable dimension. Consider also that each individual control input has known saturation bounds

$$
\left|e_{j} u_{k}\right| \leq \mu_{j}, \quad \mu_{j} \in \mathbb{R}, \quad j: 1, \ldots, c
$$

where $e_{j}$ is the standard canonical row vector in $\mathbb{R}^{c}$ whose $j$-th component is one and the rest are zero. Hence, a set of vectors $\overline{u_{i}}, i: 1, \ldots, 2^{c}$ can be constructed such that the control action $u$ belongs to its convex hull.

Theorem 1. Assume $\left\{x_{0}, x_{-1}\right\} \in \Phi$. Then, the system (3) with the control law (12) is locally stable in region (14), satisfies the control input saturation (16) and $\Phi$ belongs to the domain of attraction of the origin if the following SOS problem is feasible for all $i: 1, \ldots, 2^{c}$ and $j: 1, \ldots, c:$

$$
\left[\begin{array}{cc}
Q\left(E x_{k}, x_{k-1}\right) & (*)^{T} \\
\Psi\left(x_{k}, x_{k-1}\right) & Q\left(E \cdot A\left(x_{k}\right) z_{k}, x_{k}\right)
\end{array}\right]-\epsilon I-\Upsilon_{1 i} \in \sum_{x_{k}, x_{k-1}}^{2 m}
$$




$$
\begin{gathered}
Q\left(E x_{k}, x_{k-1}\right)-\epsilon I-W_{1}-\mathcal{H}_{1} \in \Sigma_{x_{k}, x_{k-1}}^{m} \\
{\left[\begin{array}{cc}
Q\left(E x_{k}, x_{k-1}\right) & (*)^{T} \\
e_{j} M\left(x_{k}, x_{k-1}\right) & \mu_{j}^{2}
\end{array}\right]-\Upsilon_{2 i} \in \Sigma_{x_{k}, x_{k-1}}^{m+1}} \\
R^{2} U^{-1}-Q\left(E x_{k}, x_{k-1}\right)-W_{2}-\mathcal{H}_{2} \in \Sigma_{x_{k}, x_{k-1}}^{m} \\
{\left[\begin{array}{cc}
\beta^{-2} z_{k}^{T} Y z_{k} & z_{k}^{T} \\
Z_{k} & Q\left(E x_{k}, x_{k-1}\right)
\end{array}\right]-\Upsilon_{3 i} \in \Sigma_{x_{k}, x_{k-1}}^{m+1}}
\end{gathered}
$$

where

$$
\begin{gathered}
\Psi\left(x_{k}, x_{k-1}\right)=T\left(E \cdot A\left(x_{k}\right)\right)\left(A\left(x_{k}\right) Q\left(E x_{k}, x_{k-1}\right)-B\left(x_{k}\right) M\left(x_{k}, x_{k-1}\right)\right), \\
\Upsilon_{d i}=S_{d i}+\mathcal{H}_{d i}+\sum_{b=1}^{n-L} H_{d b} \phi_{b i}, \quad d: 1, \ldots, 3
\end{gathered}
$$

being $\epsilon>0$ and:

$$
\begin{aligned}
& \tilde{\phi}_{b}=e_{b}\left(E x_{k}-E \cdot A\left(x_{k-1}\right) z_{k-1}\right), \\
& \phi_{b i}=e_{b}\left(E^{\perp} x_{k}-E^{\perp} A\left(x_{k-1}\right) z_{k-1}+E^{\perp} B\left(x_{k-1}\right) \bar{u}_{i}\right), \\
& W_{1} \in \wp^{m}\left(R^{2}-z_{k}^{T} U z_{k}, R^{2}-z_{k-1}^{T} U z_{k-1}\right), W_{2} \in \wp^{m}\left(R^{2}-z_{k-1}^{T} U z_{k-1}\right), \\
& S_{1 i} \in \wp^{2 m}\left(R^{2}-z_{k}^{T} U z_{k}, R^{2}-z_{k-1}^{T} U z_{k-1}\right), \\
& S_{2 i} \in \wp^{m+1}\left(R^{2}-z_{k}^{T} U z_{k}, R^{2}-z_{k-1}^{T} U z_{k-1}\right), S_{3 i} \in \wp^{m+1}\left(\beta^{2}-z_{k-1}^{T} Y z_{k-1}\right), \\
& \mathcal{H}_{1} \in \mathcal{J}^{m}\left(\tilde{\phi}_{1}, \ldots, \tilde{\phi}_{L}\right), \mathcal{H}_{2} \in \mathcal{J}^{m}\left(R^{2}-z_{k}^{T} U z_{k}, \tilde{\phi}_{1}, \ldots, \tilde{\phi}_{L}\right), \\
& \mathcal{H}_{1 i} \in \mathcal{J}^{2 m}\left(\tilde{\phi}_{1}, \ldots, \tilde{\phi}_{L}\right), \quad\left\{\mathcal{H}_{2 i}, \mathcal{H}_{3 i}\right\} \in \mathcal{J}^{m+1}\left(\tilde{\phi}_{1}, \ldots, \tilde{\phi}_{L}\right), \\
& H_{1 b} \in \mathcal{R}_{x_{k}, x_{k-1}}^{(2 m) \times(2 m)},\left\{H_{2 b}, H_{3 b}\right\} \in \mathcal{R}_{x_{k}, x_{k-1}}^{(m+1) \times(m+1)} .
\end{aligned}
$$

The decision variables in the above problem are the coefficients (note that degrees are chosen beforehand) of the polynomial matrices 
$W_{1}, W_{2}, S_{1 i}, S_{2 i}, S_{3 i}, \mathcal{H}_{1}, \mathcal{H}_{2}, \mathcal{H}_{1 i}, \mathcal{H}_{2 i}, \mathcal{H}_{3 i}, H_{1 b}, H_{2 b}, H_{3 b}, \quad Q\left(E x_{k}, x_{k-1}\right) \quad$ and $M\left(x_{k}, x_{k-1}\right)$.Proof: Using the candidate Lyapunov function (11), stability condition (8) now becomes:

$$
\Delta V=z_{k+1}^{T} Q^{-1}\left(\tilde{x}_{k+1}, x_{k}\right) z_{k+1}-z_{k}^{T} Q^{-1}\left(\tilde{x}_{k}, x_{k-1}\right) z_{k}<0
$$

Substituting $z_{k+1}$ by its value

$$
z_{k+1}=T\left(E \cdot A\left(x_{k}\right)\right)\left(A\left(x_{k}\right)-B\left(x_{k}\right) K\left(x_{k}, x_{k-1}\right)\right) z_{k},
$$

performing the well-known change of variable

$$
\rho=Q^{-1}\left(\tilde{x}_{k}, x_{k-1}\right) z_{k}
$$

and applying Schur complement, it leads to

$$
\eta^{\mathrm{T}}\left[\begin{array}{cc}
Q\left(\tilde{x}_{k}, x_{k-1}\right) & (*)^{T} \\
\Psi\left(x_{k}, x_{k-1}\right) & Q\left(\tilde{x}_{k+1}, x_{k}\right)
\end{array}\right] \eta \geq 0
$$

being $\eta$ a vector of independent variables.

The relationship between present and past states is:

$$
E^{\perp}\left(x_{k}-A\left(x_{k-1}\right) z_{k-1}-B\left(x_{k-1}\right) u_{k-1}\right)=0, E\left(x_{k}-A\left(x_{k-1}\right) z_{k-1}\right)=0
$$

This information can be introduced in the SOS constraints with terms $\mathcal{H}\left(x_{k}, x_{k-1}\right)$ belonging to the ideals associated to the above equalities. However, in order to avoid introducing new variables $u$ in the SOS program, equalities in (24) depending on $E^{\perp}$ must be introduced with arbitrary multiplier matrices $H_{d b}\left(x_{k}, x_{k-1}\right)$, conforming the rightmost summation in the definition of $\Upsilon_{d i}$ in (22), but keeping linearity in $\phi_{b i}$. In fact, to actually get (22), a last step is needed: as the resulting expressions are affine in $u_{k-1}$, they will hold if they do in all the vertices given by vectors $\bar{u}_{i}$, from convexity argu- 
ments. Note that multipliers $H_{d b}$ must be shared between all vertices.

Now, positive semi-definite matrix multipliers $W_{i}\left(x_{k}, x_{k-1}\right), S_{d i}\left(x_{k}, x_{k-1}\right)$ are provided in order to add information about $\Omega$ in SOS conditions so that they need to hold only locally (note that multipliers $S_{d i}$ can actually be different for different $\bar{u}_{i}$ ). After these steps, (17) and (18) are obtained, so (11) is a valid Lyapunov function as (6)-(8) hold locally in $\Omega$.

Define now $\Theta$ as the Lyapunov level set

$$
\Theta=\left\{x_{k}, x_{k-1}: V\left(x_{k}, x_{k-1}\right) \leq 1\right\}
$$

Conditions (19) ensure that $u$ does not take values larger than the saturation bounds $\mu$ inside the region $\Pi=\Theta \cap \Omega$. They are obtained from the inequality

$$
z_{k}^{T} Q^{-1}\left(\tilde{x}_{k}, x_{k-1}\right) z_{k}-z_{k}^{T} e_{j}^{T} K\left(x_{k}, x_{k-1}\right)^{T} \mu_{j}^{-1} I(*) \geq 0
$$

in a similar way to the quadratic case (Boyd 1994) for $\Theta$, but relaxed with local information on $\Omega$ and system dynamics analogous to the above discussed multipliers ${ }^{2}$.

As a last step in the proof, as locality conditions only hold in $\Omega$, we need to ensure that there exists an invariant subset of $\Omega$ containing the initial set $\Phi$.

Let us assume $V\left(x_{k}, x_{k-1}\right) \geq 1 \forall x_{k} \in \partial \Omega_{0}, x_{k-1} \in \Omega_{0}$ which is enforced by (20) as later shown. Let us prove that $\Pi=\Theta \cap \Omega$ is invariant. Indeed, the points $x_{k} \in \partial \Omega_{0}$ and $x_{k-1} \in \Omega_{0}$ are outside $\Pi$, so the trajectories will never leave $\Pi$ through that part of $\partial \Omega$.

${ }^{2}$ Actually, we should prove $\mu_{j}-z e_{j}^{T} K^{T} K e_{j} z>0$ via multipliers in the cone $\left(1-z^{T} Q^{-1} z\right)$ and the rest of constraints defining (14) and system dynamics (24). However, the need of the change of variable (23) forces the use of some constant (S-procedure like) multipliers because relationship between $\rho$ and $x$ is lost (details omitted for brevity). 
If $x_{k} \in \Omega_{0}, x_{k-1} \in \partial \Omega_{0}, V\left(x_{k}, x_{k-1}\right) \leq 1$ then $x_{k+1} \in \Omega_{0}, x_{k} \in \Omega_{0}$ and $V\left(x_{k+1}, x_{k}\right)<$ 1. Indeed, $V\left(x_{k+1}, x_{k}\right)<1$ from (17); then expression (20), from the above paragraph, discards the option of $x_{k+1}$ leaving $\Omega_{0}$. Hence, if $\left\{x_{k}, x_{k-1}\right\} \in \Pi$, we have $\left\{x_{k+1}, x_{k}\right\} \in$ $\Pi$.

To enforce $V\left(x_{k}, x_{k-1}\right) \geq 1 \forall x_{k} \in \partial \Omega_{0}, x_{k-1} \in \Omega_{0}$, similar issues to those arising in (19) discussed in footnote 2 apply. Thus, resorting to similar argumentations gives (20).

The last set of SOS constraints must ensure the initial condition set $\Phi \subset \Pi$. As $\Phi \subset \Omega$ by assumption, $\Phi \subset \Theta$ has to be ensured, too. It can be proved by enforcing $V\left(x_{k}, x_{k-1}\right) \leq 1 \forall\left\{x_{k}, x_{k-1}\right\} \in \Phi$. A sufficient condition for this to hold is

$$
\frac{1}{\beta^{2}} z_{k}^{T} Y z_{k}-z_{k}^{T} Q^{-1}\left(\tilde{x}_{k}, x_{k-1}\right) z_{k} \geq 0
$$

enforced locally in $\Omega$ by (21), after applying Schur complement and Positivstellensatz. Details omitted for brevity.

So, $\left\{x_{0}, x_{-1}\right\} \in \Phi \subset \Pi \subset \Omega$, invariance of $\Pi$ has been ensured by SOS constraints and $\Pi \subset \Theta$ ensures the control action bounds (16) are met, so multipliers arising from (24) are valid.

Now, the proven invariant set in the augmented space is not a Lyapunov function level set: the level set $\Theta$ can actually extend outside the local-stability region $\Omega$, removing conservativeness. So, the discrete-time analog to La-Salle invariance theorem needs to be invoked: the system will converge to the largest invariant set in $\Delta V=0$, and only the origin verifies the zero-increment condition (details omitted for brevity).

Remark 1: With $Q\left(\tilde{x}_{k}\right), z_{k}=T\left(\tilde{x}_{k}\right) x_{k}$ and $u=-K\left(x_{k}\right) z_{k}$, Theorem 1 reduces to cases 
in Xu et al. (2007), Tanaka et al. (2008). A more general version encompassing the "natural" case $V=z_{k}^{T} Q\left(x_{k}\right) z_{k}$ may be crafted by letting $Q\left(x_{k}, x_{k-1}\right)$. In that case, the SOS problems would involve variables $\left(x_{k}, x_{k-1}, x_{k+1}\right)$. However, in order to keep convexity, new multipliers analogous to (22) are needed, with additional $\phi_{b}$ and $\phi_{b i}$ now referring to the relationship between $x_{k}$ and $x_{k+1}$. Details are omitted because the usefulness of the approach is limited, as the controller cannot depend on future $x_{k+1}$.

Remark 2: In discrete-time, Lyapunov-Krasovskii (LK) functionals are actually a particular case of generic Lyapunov functions of an augmented finite-dimensional realization incorporating delayed states (well known, for instance in Hetel et al. (2008), Gonzalez et al. (2013)). From the realization $\psi_{k}=\left(x_{k} x_{k-1}\right)^{T}$ considering a delayed controller $u_{k}=-\left(K_{1}\left(x_{k}, x_{k-1}\right) K_{2}\left(x_{k}, x_{k-1}\right)\right) \psi_{k}$, then the closed loop is:

$$
\begin{gathered}
\psi_{k+1}=\tilde{A}\left(x_{k}, x_{k-1}\right) \psi_{k} \\
\tilde{A}\left(x_{k}, x_{k-1}\right)=\left[\begin{array}{cc}
A\left(x_{k}\right)-B\left(x_{k}\right) K_{1}\left(x_{k}, x_{k-1}\right) & -B\left(x_{k}\right) K_{2}\left(x_{k}, x_{k-1}\right) \\
I & 0
\end{array}\right]
\end{gathered}
$$

If a candidate "full" Lyapunov function (encompassing any quadratic LK choices for unit delay) $V\left(x_{k}, x_{k-1}\right)=\psi^{T} Q\left(\tilde{x}_{k}, x_{k-1}\right)^{-1} \psi$ (being $Q\left(\tilde{x}_{k}, x_{k-1}\right)$ a suitably partitioned $2 \times 2$ block-polynomial matrix) is chosen, and changes of variable leading to (17) are enforced, then the Lyapunov discrete increment

$$
V_{k+1}-V_{k}=\psi_{k}^{T}\left(\tilde{A}\left(x_{k}, x_{k-1}\right)^{T} Q\left(\tilde{x}_{k+1}, x_{k}\right)^{-1} \tilde{A}\left(x_{k}, x_{k-1}\right)-Q\left(\tilde{x}_{k}, x_{k-1}\right)^{-1}\right) \psi_{k}
$$

leads to a $4 \times 4$ block-polynomial matrix condition $\Pi\left(x_{k}, x_{k-1}\right)>0$ by Schur complement (details omitted for brevity). Then, a necessary condition to ensure $\Pi\left(x_{k}, x_{k-1}\right)>$ 
0 is, taking the minor obtained from its first and third rows and columns, the following

$$
\begin{gathered}
\text { inequality: }\left[\begin{array}{cc}
Q_{11}\left(\tilde{x}_{k}, x_{k-1}\right) & (*)^{T} \\
A\left(x_{k}\right) Q_{11}\left(\tilde{x}_{k}, x_{k-1}\right)-B\left(M_{1}+M_{2}\right) & Q_{11}\left(\tilde{x}_{k+1}, x_{k}\right)
\end{array}\right]>0, \\
M_{1}=K_{1}\left(x_{k}, x_{k-1}\right) Q_{11}\left(\tilde{x}_{k}, x_{k-1}\right),
\end{gathered}
$$

which proves that the developments in this paper do not lose generality with respect to the full controllers and Lyapunov matrices above. Indeed, if $z_{k}=x_{k}\left(\operatorname{so} T\left(x_{k}\right)=I\right)$, (25) is condition (17) without Positivstellesatz terms $\Upsilon_{1 i}$. Therefore, if (25) holds with particular $M_{1}, M_{2}$, so it will with a single $M_{1}, M_{2}=0$, i.e., there will exist a single controller gain $K$ fulfilling (17). The case $T \neq I$ can also be easily set up. So, this is the motivation on why (11) is taken as a LK candidate (equivalent Lyapunov function of the augmented system) instead of other more complex constructions which would not be useful with the proposed developments.

Remark 3: Presence of $x_{k-1}$ in $Q$ instead of only $Q\left(\tilde{x}_{k}\right)$ (or $Q\left(x_{k}\right)$, remark above), allows controller $M\left(x_{k}, x_{k-1}\right)$ to take into account present and past information, so it provides more degrees of freedom to find a solution which does not violate the saturation constraints. Note also that, even if, of course, an undelayed controller $u\left(x_{k}\right)$ achieving the same performance and constraints will likely exist, maybe it cannot be obtained with convex SOS conditions.

In this approach, the bilinearity has been resolved by conceiving a full-rank matrix $\left[E E^{T}\right]$ and an implicit change of coordinates, so that:

(1) In the nullspace of $B$, we can add an arbitrary multiplier because the control action and the matrix $K$ do not appear. Also, the Lyapunov function can depend on $E x_{k}$ due to the nullification of $B$. So, no conservatism from the "delay" trick is induced in this subspace. 
(2) In the image space of $B$, in order to avoid decision variables in " $K$ ", the actual control variable must be kept. Then, as $H(u-K z)$ would be bilinear (due to the product of Positivstellensatz multiplier $H$ and controller $K$ decision variables), saturation constraints on $u$ should be added either by Positivstellensatz conditions or, as we chose, by convex-hull argumentations. This may be conservative (we are only considering bounds on $u$, considered independent of decision variables, instead of $u=K z$ ) but allows for more general Lyapunov functions and controllers which effectively achieved improved results. See the example in Section 4.

\section{Example}

Consider the following polynomial system:

$$
x_{k+1}=\left[\begin{array}{cc}
-0.7 & 0.05 \\
0.3 x_{2}\left(1-0.166 x_{1}^{2}\right) & 0.8
\end{array}\right] x_{k}+\left[\begin{array}{c}
-0.02 \\
0.05 x_{1_{k}}
\end{array}\right] u_{k}
$$

The goal will be to obtain the largest possible region of initial conditions $\Phi$, with a predefined shape, for a fixed degree in the Lyapunov function and multipliers. Given the model, as $E=0, E^{\perp}=I$, then $z_{k}=x_{k}$ is the only option.

Conditions to find a global controller with a quadratic $V\left(x_{k}\right)$, i.e., constant $Q$, are infeasible. Note that setting a polynomial $Q\left(\tilde{x}_{k}\right)$ is not a viable option, as $E=0$, so $\tilde{x}_{k}$ is empty.

Now define, for instance, a spherical state-space region of interest $\Omega$ and a spherical region of initial conditions $\Phi$ as:

$$
\Omega=\max \left(x_{1_{k}}^{2}+x_{2_{k}}^{2}, x_{1_{k-1}}^{2}+x_{2_{k-1}}^{2}\right) \leq 4.5^{2}
$$




$$
\Phi=\max \left(x_{1_{k}}^{2}+x_{2_{k}}^{2}, x_{1_{k-1}}^{2}+x_{2_{k-1}}^{2}\right) \leq \beta^{2}
$$

The objective will be maximizing the size parameter $\beta$ for fixed control action bounds $\mu,\left|u_{k}\right| \leq \mu$, while proving that $\Phi$ belongs to the domain of attraction of the origin, enforcing the existence of a Lyapunov level set larger than $\Phi$ included in $\Omega$. The maximum degree for $M\left(x_{k}, x_{k-1}\right)$ and $Q\left(x_{k-1}\right)$ is set to two. The parameterizations of Positivstellensatz terms are:

$$
\begin{aligned}
& W_{1}=\psi_{1}\left(4.5^{2}-x_{1_{k-1}}^{2}-x_{2_{k-1}}^{2}\right), \quad W_{2}=\psi_{2}\left(4.5^{2}-x_{1_{k-1}}^{2}-x_{2_{k-1}}^{2}\right), \\
& S_{d i}=\varrho_{d i}\left(4.5^{2}-x_{1_{k}}^{2}-x_{2_{k}}^{2}\right)+\psi_{d i}\left(4.5^{2}-x_{1_{k-1}}^{2}-x_{2_{k-1}}^{2}\right), \quad d: 1,2
\end{aligned}
$$$$
S_{3 i}=\psi_{3 i}\left(\beta^{2}-x_{1_{k-1}}^{2}-x_{2_{k-1}}^{2}\right) \text { and } \mathcal{H}_{1}=\mathcal{H}_{2}=0 \text {, }
$$

where $\psi_{1}, \psi_{2}, \psi_{d i}$ and $\varrho_{d i}$ are diagonal matrices of appropriate dimension whose entries belong to $\Sigma_{x_{k}, x_{k-1}}$, with a maximum degree of 4 . Similarly, terms $H_{d i}$ in Theorem 1 are taken again as diagonal matrices whose entries belong to $\mathcal{R}_{x_{k}, x_{k-1}}$ of degree 4 .

Once the problem is set up, constant Lyapunov functions from literature are compared to the delayed approach here proposed. Feasible solutions were found by software SOSOPT using the image representation of the SOS problem (Balas et al. 2012, Seiler et al. 2013).

The largest $\beta$ obtained until infeasibility with the different approaches is shown in Table 1. Row 1 presents results with constant decision variables $Q, M$; row 2 presents results obtained using Xu et al. (2007) approach, row 3 present results with more flexible parameterizations allowed by Theorem 1 . 
Table 1. Comparison of different approaches

\begin{tabular}{l||c|c|c}
\multicolumn{1}{c||}{$\beta$} & $\mu=\infty$ & $\mu=6.3$ & $\mu=1.05$ \\
\hline \hline$Q, M$ & Inf & Inf & Inf \\
\hline$Q, M\left(x_{k}\right)$ & 1.273 & 1.272 & 0.937 \\
\hline$Q\left(x_{k-1}\right)$, & 1.275 & 1.383 & 1.162 \\
$M\left(x_{k}, x_{k-1}\right)$ & & &
\end{tabular}

* Inf $\equiv$ infeasible

Table 2 shows the amount of RAM memory, the time spent in the parsing phase and the time employed by the solver to obtain a solution for each of the considered approaches with $\mu=6.3$ (i.e., to compute the figures in the center column of Table 1 ). The code was executed in an Intel ${ }^{\circledR}$ Core $^{\mathrm{TM}} 2$ Duo CPU P8600 2.4GHz, 4 Gb DDR3 RAM machine running MATLAB R2011b with SOSOPT 2.01 and SeDuMi 1.3.

Table 2. Approximate computational resources with the different approaches.

\begin{tabular}{l||c|c|c|c} 
& Problem Size & RAM & Parser Time & Solver Time \\
\hline$Q, M$ & $774 \times 201$ & $10 \mathrm{Mb}$ & $1.19 \mathrm{~s}$ & $0.96 \mathrm{~s}$ \\
\hline$Q, M\left(x_{k}\right)$ & $784 \times 201$ & $10 \mathrm{Mb}$ & $1.08 \mathrm{~s}$ & $0.76 \mathrm{~s}$ \\
\hline$Q\left(x_{k-1}\right)$, & $21041 \times 3320$ & $230 \mathrm{Mb}$ & $25.84 \mathrm{~s}$ & $65.26 \mathrm{~s}$ \\
$M\left(x_{k}, x_{k-1}\right)$ & & &
\end{tabular}

Looking at Table 1, it can be seen that a linear controller cannot be proven to stabilize the system in region $\Omega$. Then, a polynomial controller $M\left(x_{k}\right)$, using Xu et al. (2007), keeps obtaining the same $\beta$ for any $6.3<\mu<\infty$.

The last row shows that improvement with respect to Xu et al. (2007) has been achieved with rational controllers arising from Theorem $1(8.73 \%$ increase of $\beta$ with $\mu=6.3$ and $24 \%$ with a 6 times lower bound $\mu=1.05$ ). Analyzing the results, it is shown that, without saturation constraints $(\mu=\infty)$, there is not enough information between past and present states, so there is practically no improvement over prior literature results. On the other hand, if saturation bound is low (rightmost column), the percent improvement over previous work is high. However, the proved region remains small because 
there is not enough input power to stabilize the system from initial conditions far away from the origin.

Note that the reported improvements come at the expense of a significantly increased computational cost: the second row considers SOS problems in $x_{k}$, the last row doubles the number of independent variables and increases the number of multipliers.

\section{Conclusions}

This paper develops a convex stabilization design for polynomial systems, which reduces some sources of conservatism in previous literature results. An extension from the classical polynomial Lyapunov function is given, based on including delayed states and knowledge about limits on the control input. The percentage improvement in performance with respect to prior results increases as input bounds get smaller. The input bound can be actually considered as a design parameter, with a maximum value given by actual physical saturation limits.

\section{Acknowledgements}

The research work by J.L. Pitarch and A. Sala has been partially supported by the Spanish government under research project DPI2011-27845-C02-01 (MINECO), and Generalitat Valenciana grant PROMETEOII/2013/004. T.M. Guerra and J. Lauber gratefully acknowledge the support of the International Campus on Safety and Intermodality in Transportation, the European Community, Délégation Régionale à la Recherche et à la Technologie, Ministère de l'Enseignement supérieur et de la Recherche, Région Nord Pas de Calais and the Centre National de la Recherche Scientifique. 


\section{References}

Balas, G., Packard, A., Seiler, P., and Topcu, U., 2012. Robustness Analysis of Nonlinear Systems [online]. Available from: http://www.aem.umn.edu/ AerospaceControl/.

Bernussou, J., Peres, P.L.D., and Geromel, J.C., 1989. A linear programming oriented procedure for quadratic stabilization of uncertain systems. Systems \& Control Letters, 13 (1), 65-72.

Boyd, S., 1994. Linear matrix inequalities in system and control theory. Philadelphia: Society for Industrial and Applied Mathematics.

Chesi, G., 2010. LMI Techniques for Optimization Over Polynomials in Control: A Survey. IEEE Transactions on Automatic Control, 55 (11), 2500-2510.

Chesi, G., 2011. Domain of Attraction Analysis and Control Via Sos Programming. Springer Verlag.

Fukuda, M. and Kojima, M., 2001. Branch-and-cut algorithms for the bilinear matrix inequality eigenvalue problem. Computational Optimization and Applications, 19 (1), 79-105.

Gassara, H., Hajjaji, A.E., Kchaou, M., and Chaabane, M., 2014. Robust $H$ o reliable control of time delay nonlinear systems via Takagi-Sugeno fuzzy models. International Journal of Systems Science, 45 (3), 667-681, (Online Sep. 2012).

Gonzalez, A., Sala, A., Garcia, P., and Albertos, P., 2013. Robustness analysis of discrete predictor-based controllers for input-delay systems. International Journal of Systems Science, 44 (2), 232-239.

Guerra, T.M., Kerkeni, H., Lauber, J., and Vermeiren, L., 2012. An Efficient Lyapunov Function for Discrete TS Models: Observer Design. IEEE Transactions on Fuzzy Systems, 20 (1), 187-192.

Hetel, L., Daafouz, J., and Iung, C., 2008. Equivalence between the LyapunovKrasovskii functionals approach for discrete delay systems and that of the stability conditions for switched systems. Nonlinear Analysis: Hybrid Systems, 2 (3), $697-705$.

Jarvis-Wloszek, Z., Feeley, R., Tan, W., Sun, K., and Packard, A., 2005. Control applications of sum of squares programming. Positive Polynomials in Control.

Khalil, H., 2002. Nonlinear systems. 3rd ed. Upper Saddle River N.J.: Prentice Hall. 
Koshkouei, A.J. and Burnham, K.J., 2011. Adaptive backstepping sliding mode control for feedforward uncertain systems. International Journal of Systems Science, 42 (12), 1935-1946.

Löfberg, J., 2009. Pre- and Post-Processing Sum-of-Squares Programs in Practice. IEEE Transactions on Automatic Control, 54, 1007-1011.

Papachristodoulou, A. and Prajna, S., 2005. Analysis of Non-polynomial Systems Using the Sum of Squares Decomposition. In: Positive Polynomials in Control. Springer Berlin Heidelberg, 23-43.

Pitarch, J.L., Sala, A., and Ariño, C.V., 2013. Closed-form estimates of the domain of attraction for nonlinear systems via fuzzy-polynomial models. IEEE Transactions on Systems, Man and Cybernetics, Part B (Cybernetics), DOI: 10.1109/TCYB.2013.2258910.

Pozo, F. and Rodellar, J., 2010. Robust stabilisation of polynomial systems with uncertain parameters. International Journal of Systems Science, 41 (5), 575-584.

Prajna, S., Papachristodoulou, A., and Wu, F., 2004. Nonlinear control synthesis by sum of squares optimization: A Lyapunov-based approach. In: Control Conference, 2004. 5th Asian. 157-165.

Sala, A. and Ariño, C., 2009. Polynomial Fuzzy Models for Nonlinear Control: A Taylor Series Approach. IEEE Transactions on Fuzzy Systems, 17 (6), 1284-1295.

Scherer, C.W. and Hol, C.W.J., 2005. Matrix Sum-of-Squares Relaxations for Robust Semi-Definite Programs. Mathematical Programming, 107 (1-2), 189-211.

Seiler, P., Zheng, Q., and Balas, G., 2013. Simplification Methods for Sum-of-Squares Programs. Cornell University Library.

Slotine, J.-J.E., 1991. Applied nonlinear control. Englewood Cliffs, N.J: Prentice Hall.

Stengle, G., 1974. A nullstellensatz and a positivstellensatz in semialgebraic geometry. Mathematische Annalen, 207 (2), 87-97.

Tanaka, K., Ohtake, H., and Wang, H.O., 2008. A SOS-based stable control of polynomial discrete fuzzy systems. In: 2008 American Control Conference. Seattle, Washington, USA: IEEE, 4875-4880.

Valmorbida, G., Tarbouriech, S., and Garcia, G., 2013. Design of Polynomial Control Laws for Polynomial Systems Subject to Actuator Saturation. IEEE Transactions on Automatic Control, 58 (7), 1758-1770. 
Xu, J., Xie, L., and Wang, Y., 2007. Synthesis of Discrete-time Nonlinear Systems: A SOS Approach. In: American Control Conference, 2007. ACC '07. Singapore: IEEE, 4829-4834.

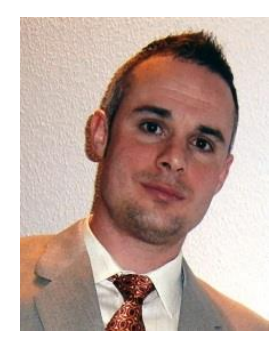

José Luis Pitarch was born in Castellón, Spain, in 1983. He received the M.Sc. degree in Industrial Engineering with honors in 2008 at Jaume I University of Castellón (SPAIN). He was with BP Oil Refinery of Castellón as process control engineer in 2009. He received the M.Sc. degree in Control and Industrial Computing in 2010 and the Ph.D. degree in Control Engineering with honors in 2013, both at Polytechnical University of Valencia (SPAIN). He is the author or coauthor of 9 conference papers and 4 journal papers. His research interests in control engineering focus in Takagi-Sugeno and fuzzy polynomial systems, using LMI/SOS approaches to perform stability analysis, with and without disturbances (domain of attraction and inescapable set estimation), nonlinear control and observer synthesis.

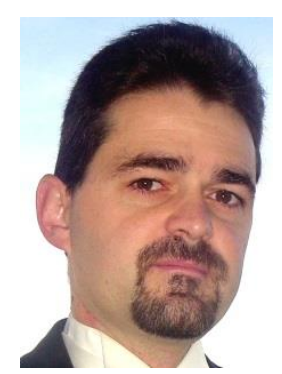

Antonio Sala was born in Valencia, Spain, in 1968. He received the B.Eng. Honours degree in Combined Engineering in 1990 at Coventry University (UK), the M.Sc Degree in Electrical Engineering in 1993, and his Ph.D. in Control Engineering in 1998 both from UPV (Valencia Technical University, Spain). He is chair professor in UPV at the Systems and Control Engineering Department since 2009. He has been teaching for more than 20 years in a wide range of subjects in the area, such as linear systems theory, multivariable process control and intelligent control. He has supervised 7 Ph.D. thesis and 30 M.Sc. final projects. He has taken part in research and mobility projects funded by local industries, government and European community. He has published more than 60 journal papers and more than 120 conference ones. In the past, he has been member of the IFAC Publications committee. Currently, he serves as Associate editor of IEEE Transactions on Fuzzy Systems and Area Editor of Fuzzy Sets and Systems. Further details at http://personales.upv.es/asala . 


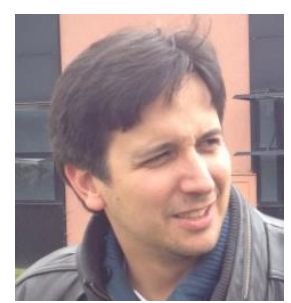

Jimmy Lauber was born in Tours, France in 1976. He is currently associated professor at the University of Valenciennes et du HainautCambrésis (UVHC), France. He received his Ph.D degree in automatic control from the UVHC in 2003 and the HDR in 2011. His major research fields are quasi-LPV (Takagi-Sugeno) models control and observation, Non quadratic Lyapunov functions... and applications to automotive powertrain management (IC engine and high downsizing, Single and dual clutch, ...).

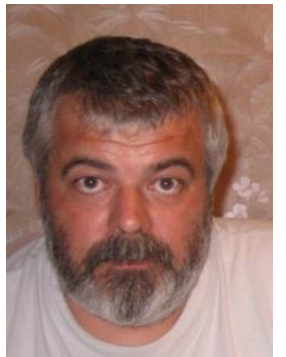

Thierry Marie Guerra was born in Mulhouse, France in 1963. He is currently full professor at the University of Valenciennes et du Hainaut-Cambrésis (UVHC), France. He received his Ph.D degree in Automatic Control from the UVHC in 1991 and the HDR in 1999. He is head of the Laboratory of Industrial and Human Automation, Mechanics and Computer Science (LAMIH CNRS UMR 8201) (100 researchers and staff, 80 $\mathrm{PhD}$ students and post-docs). He is vice-chair of the Technical Committee 3.2 "Computational Intelligence in Control" for IFAC (International Federation of Automatic Control), member of the IFAC TC 7.1 "Automotive Control", Area Editor of the international journals Fuzzy Sets \& Systems and IEEE Transactions on Vehicular Technology. His major research fields and topics of interest are, wine, hard rock, chess, nonlinear control, LPV, quasi-LPV (Takagi-Sugeno) models control and observation, LMI constraints, Non quadratic Lyapunov functions... and applications to powertrain systems (IC engine, electrical motors, hybrid vehicles, fuel cells ...) and disabled persons. 